Open Access

\title{
Gene-expression analysis of adult-onset Still's disease and systemic juvenile idiopathic arthritis is consistent with a continuum of a single disease entity
}

Nanguneri Nirmala ${ }^{1}$, Arndt Brachat ${ }^{2}$, Eugen Feist ${ }^{3}$, Norbert Blank ${ }^{4}$, Christof Specker $^{5}$, Matthias Witt ${ }^{6}$, Jan Zernicke ${ }^{3}$, Alberto Martini ${ }^{7}$ and Guido Junge ${ }^{8^{*}}$

\begin{abstract}
Background: Adult-onset Still's disease (AOSD), a rare autoinflammatory disorder, resembles systemic juvenile idiopathic arthritis (SJIA). The superimposable systemic clinical features of AOSD and SJIA suggest both clinical phenotypes represent the same disease continuum with different ages of onset. To further characterize the similarity between AOSD and SJIA at the molecular level, 2 previously identified response gene sets in SJIA were used to investigate how genes that respond to interleukin (IL)-1 $1 \beta$ inhibition with canakinumab in SJIA patients behave in AOSD patients with active disease prior to IL-1 $\beta$ targeting therapy, relative to healthy subjects.
\end{abstract}

Findings: All genes downregulated in SJIA patients following canakinumab treatment were upregulated in most patients with active AOSD prior to canakinumab treatment, relative to healthy subjects. A few patients with milder AOSD had expectedly gene-expression patterns that resembled those in healthy subjects. Comparison of the gene-expression patterns with neutrophil counts showed a correlation between elevated neutrophil numbers and upregulation of canakinumab-responsive genes. Correspondingly, most genes upregulated following canakinumab treatment in patients with SIIA patients were downregulated in the majority of AOSD patients.

Conclusions: These results further support the concept of a Still's disease continuum that includes both a pediatric/ juvenile onset (SJIA) and adult onset (AOSD) form.

Keywords: Adult-onset Still's disease, Canakinumab, Gene expression, Interleukin-1 $\beta$, Systemic juvenile idiopathic arthritis

\section{Findings}

Background and research hypothesis

Adult-onset Still's disease (AOSD) is a rare autoinflammatory disorder resembling the pediatric syndrome systemic juvenile idiopathic arthritis (SJIA) [1, 2]. Patients with either AOSD or SJIA exhibit classical clinical and laboratory features, including daily spiking fever, arthralgia or arthritis, evanescent rash, and increased white blood cell count (mainly neutrophils) [1, 3-5]. Because of the superimposable systemic clinical features of SJIA and AOSD, it has been suggested that these clinical phenotypes represent the same disease continuum with different ages of onset.

\footnotetext{
* Correspondence: guido.junge@novartis.com

${ }^{8}$ Novartis Pharma AG, CH-4002 Basel, Switzerland

Full list of author information is available at the end of the article
}

It has, therefore, been proposed that patients with the systemic features of SJIA but without arthritis be classified as having Still's disease based on analogy with AOSD [2]. Of note, when children were first described in the $19^{\text {th }}$ century with what is now called "SJIA," the term "Still's disease" was used [6].

In addition to having a similar clinical picture, evidence suggests that AOSD and SJIA are also comparable on a molecular level. Both conditions are characterized by activation of the innate immune system, including elevations of inflammatory cytokines and proteins such as interleukin (IL)-1, IL-6, IL-18, and S100 proteins [7-11]. Moreover, patients with AOSD or SJIA can experience macrophage activation syndrome, a severe, potentially life-threatening complication $[12,13]$.

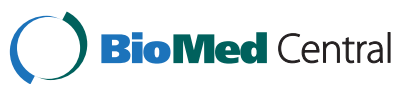

(c) 2015 Nirmala et al. Open Access This article is distributed under the terms of the Creative Commons Attribution 4.0 International License (http://creativecommons.org/licenses/by/4.0/), which permits unrestricted use, distribution, and reproduction in any medium, provided you give appropriate credit to the original author(s) and the source, provide a link to the Creative Commons license, and indicate if changes were made. The Creative Commons Public Domain Dedication waiver (http://creativecommons.org/publicdomain/zero/1.0/) applies to the data made available in this article, unless otherwise stated. 
The most compelling evidence demonstrating that AOSD and SJIA largely share the same pathophysiology may be that both are highly responsive to IL-1 inhibition. Translational and clinical studies suggest that the proinflammatory cytokine IL-1 plays a pivotal role in the pathogenesis of SJIA. Serum samples from patients with SJIA have been shown to induce the transcription of innate immunity genes, including IL-1, in healthy peripheral blood mononuclear cells [14]. In addition, these cells in patients with SJIA demonstrated a possible IL-1-driven signature [15]. More importantly, the pivotal role of IL-1 in the pathogenesis of SJIA has been validated by the clinical efficacy of canakinumab - a high-affinity human monoclonal antiIL-1 $\beta$ antibody - in phase 2 and 3 SJIA trials [16, 17]. Evidence for the clinical efficacy of IL-1 inhibition in adult patients with moderate to severe AOSD, including those resistant to standard therapy, is principally derived from small observational studies [10, 18-22]. Further, results from 1 open-label, randomized trial suggest that IL-1 inhibitor therapy is effective in AOSD and may minimize or avoid the need for glucocorticoids in most patients [23, 24].

Analyses of gene-expression profiles can be clinically useful not only for disease classification, diagnosis, and prognosis but also to identify disease-specific treatment effects that target underlying pathologic mechanisms. In a previous gene-expression analysis using blood samples from patients with SJIA treated with canakinumab in phase 3 trials, canakinumab strongly repressed many inflammation- and innate-immunity-related genes, including those in the IL-1-signaling pathways, in canakinumab responders [25]. To evaluate if AOSD is also an IL-1 - driven condition, the present study investigated how genes that respond to IL-1 $\beta$ inhibition with canakinumab in patients with SJIA behave in patients with active AOSD, relative to healthy subjects. It was hypothesized that because AOSD and SJIA are closely related conditions, genes that respond to canakinumab treatment in patients with SJIA may be inversely dysregulated in patients with AOSD prior to anti - IL-1 $\beta$ therapy. If this proved to be the case, then a similar set of genes in AOSD should be responsive to anti - IL1- $\beta$ therapy.

\section{Patients and methods}

In the previous SJIA gene-expression analysis, pre- and post-canakinumab treatment blood samples were obtained from patients participating in two phase 3 clinical trials ( $\beta$-SPECIFIC- 1 and $\beta$-SPECIFIC-2) [25]. To identify canakinumab-responsive genes, baseline (pretreatment) and day-3 (post-treatment) samples were compared from patients who clearly benefited from canakinumab treatment by achieving a $\geq 50 \%$ improvement by adapted pediatric American College of Rheumatology criteria at

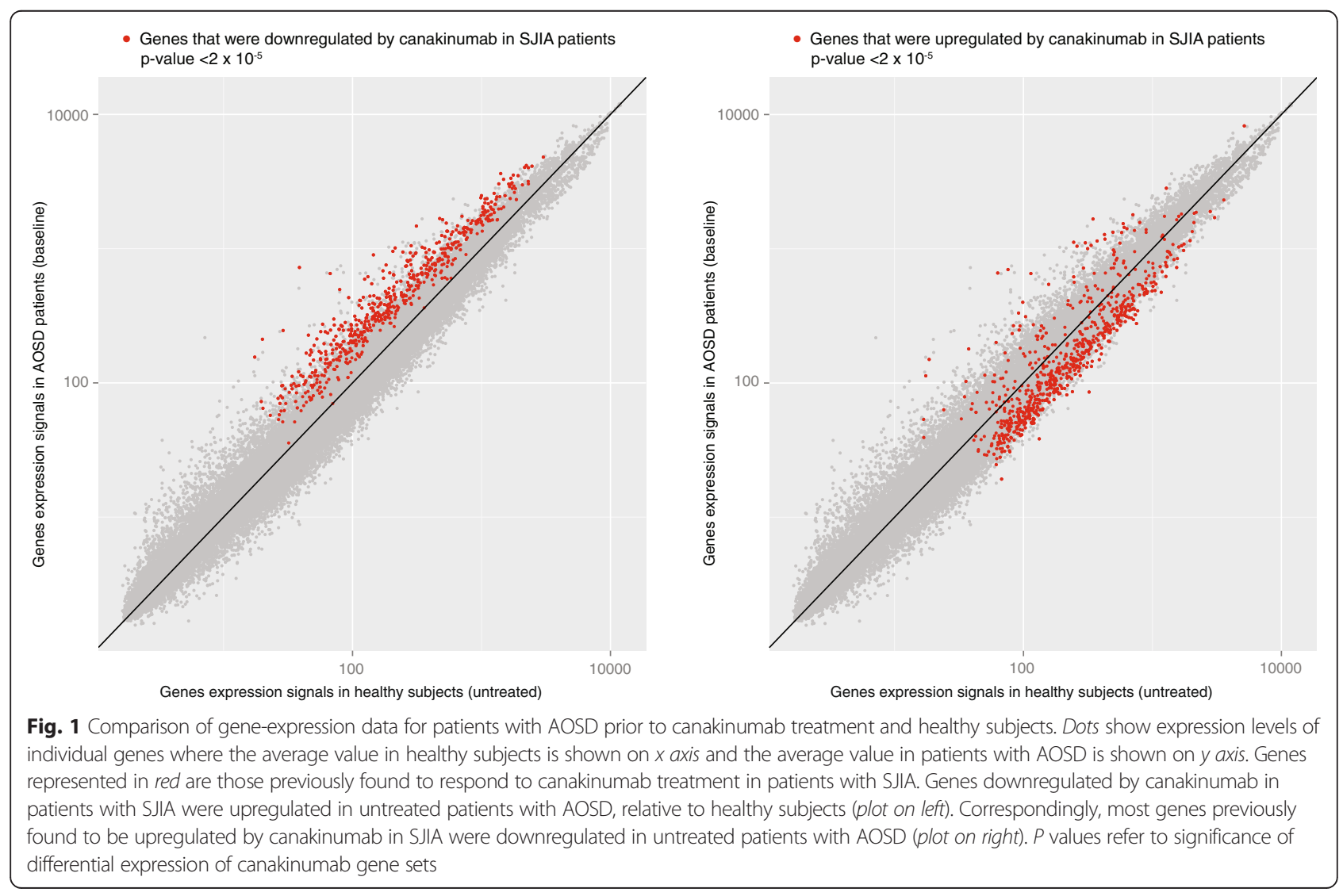


day 15 (median age, 9 years). The canakinumab-response signature in SJIA included genes that were significantly differentially expressed (paired $t$-test: $P<.05 ; \geq 1.5$-fold differential expression) at day 3 compared with baseline, represented by 577 downregulated probe sets and 728 upregulated probe sets.

For the present gene-expression analysis in AOSD, blood samples were obtained from 17 patients (median age, 37 years; $59 \%$, female) under investigation for the efficacy of canakinumab in patients with active AOSD [NCT02204293] [26]. Blood samples were also obtained from 19 healthy subjects included in the control group (median age, 26 years; $79 \%$, female). The probe sets identified in the blood samples of patients with SJIA were used for supervised visualization of gene-expression values in the untreated patients with AOSD and healthy subjects. The data were median-centered per gene to visualize the direction of differential expression more clearly.

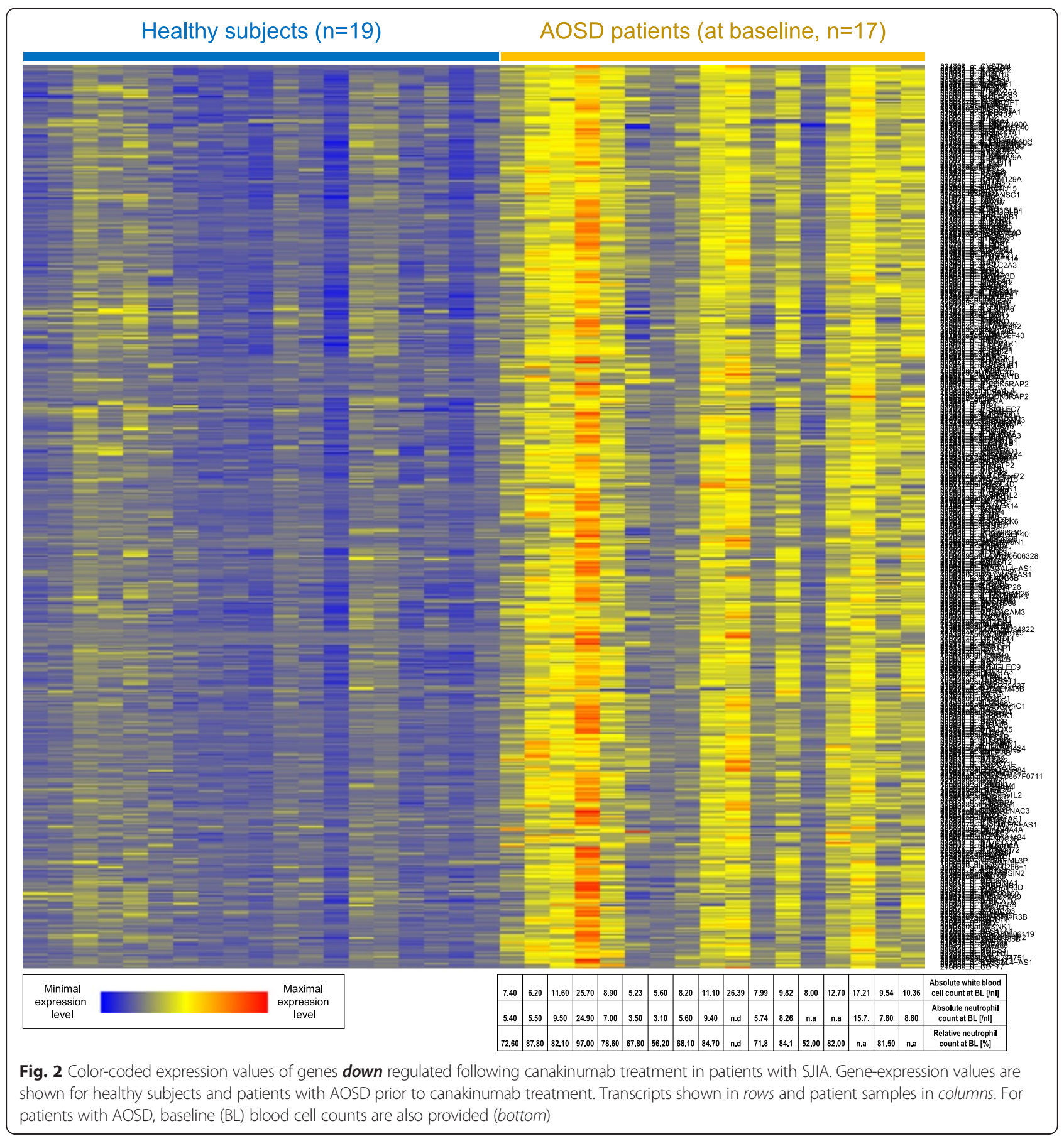


Whole blood samples were collected in PAXgene Blood RNA tubes (Qiagen, Hilden, Germany) and stored at $-80^{\circ}$ C. Total RNA was subsequently isolated with the PAXgene Blood RNA Kit (Qiagen). The synthesis of cDNA was performed using the Ovation ${ }^{\circledR}$ RNA Amplification System V2 including the Ribo-SPIA ${ }^{\oplus}$ amplification process according to the instructions of the manufacturer (NuGEN Technologies Inc., San Carlos, CA). The amplification process was performed in 3 stages: (1) a 1st-strand cDNA synthesis with
oligo(dT) primers and Ovation WB Reagent (NuGEN), (2) a 2nd-strand cDNA synthesis, and (3) a single-primer, linear isothermal amplification (SPIA ${ }^{\mathrm{T}}, \mathrm{NuGEN)}$ that produced amplified single-stranded biotin-labeled cDNA. The cDNA was hybridized to GeneChip ${ }^{\circledR}$ Human Genome U133 Plus 2.0 Array as specified by the manufacturer (Affymetrix, Inc., Santa Clara, CA). Gene-expression values were stored in CEL files that were used for robust multi-array average normalization with the affy and

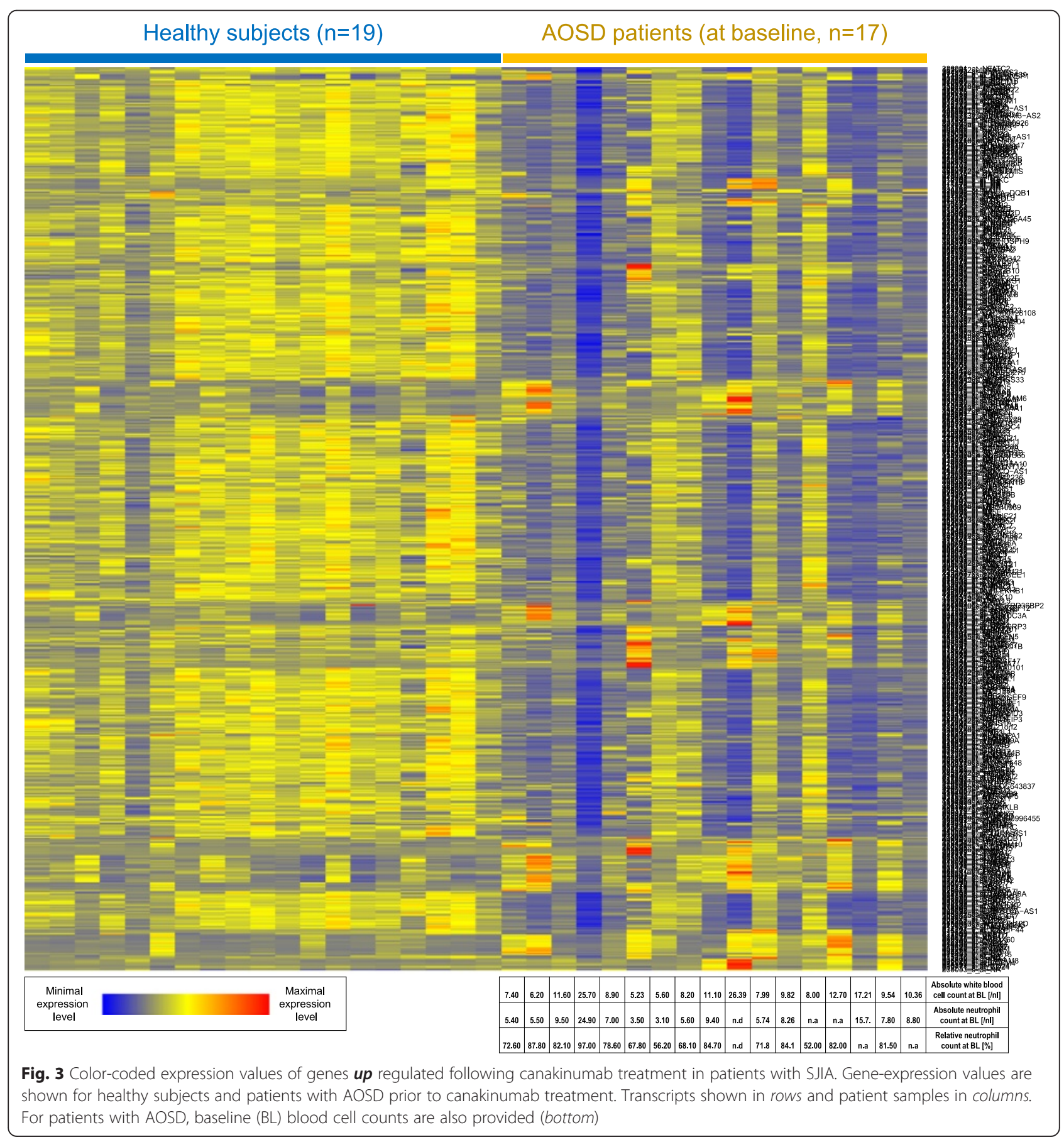


gcrma R packages. Normalized data were then scaled to a trimmed mean value of 150 . The significance of gene-set enrichment was estimated using the ROAST method as implemented in $\mathrm{R}$, [27] applying 10,000 rotations to the data set.

\section{Results and discussion}

The behavior of canakinumab responsive genes in patients with AOSD and healthy subjects is shown in Figs. 1, 2, and 3. Figure 1 displays the average expression values in the AOSD and healthy groups, whereas Figs. 2 and 3 show the relative expression values in all individuals separately. All genes that were downregulated following canakinumab treatment in patients with SJIA showed upregulation in most patients with AOSD, relative to healthy subjects (Figs. 1 and 2). These upregulated genes included various genes related to innate immunity, including several members of the IL-1-signaling pathways, e.g. IL-1 $\beta$, IL-1RAP, IL-1RN, IL-1R1, and IL-1R2. A few patients with milder AOSD had gene-expression profiles that rather resembled those of the healthy subjects (Fig. 2). Comparison of the AOSD gene-expression patterns with neutrophil counts showed that upregulation of IL-1 - associated gene expression was particularly pronounced in patients with strongly elevated neutrophil numbers and that patients with comparatively low neutrophil counts showed expression of canakinumab responsive genes at levels similar to healthy subjects. Correspondingly, most of the genes that were found to be upregulated following canakinumab treatment in patients with SJIA showed downregulation in most AOSD patients (Figs. 1 and 3), with the transcriptional patterns slightly more heterogeneous. These genes included many regulators of proliferation and immunecell activity, such as AKT3, CD24, CD28, CD3D, CD6, CD69, CDC25B, and CDC7.

While relatively new, gene expression allows analysis of hundreds and thousands of different genes at a given time instead of a handful of candidates, and represents the most advanced and comprehensive approach to screening gene activity as well as molecular networks involved in the innate immune system. Nonetheless, SJIA and AOSD are rare autoinflammatory conditions; therefore, large prospective and longitudinal trials are difficult to generate which explains the relatively small sample size of this analysis. Disease duration and severity, prior treatment with other biologics and concomitant corticosteroid exposure during the trial may have influenced the SJIA findings. With regard to the AOSD baseline analysis it should be noted that future analysis needs to confirm the hypothesized treatment response and downregulation of canakinumab responsive genes.

In conclusion, the results of this gene-expression analysis are consistent with and further support the concept of a Still's disease continuum that includes both a pediatric/ juvenile onset (SJIA) and adult onset (AOSD). These findings support further clinical evaluation of anti - IL- $1 \beta$ therapy in patients with AOSD given that AOSD appears to be an IL-1-driven condition similar to SJIA.

\section{Ethical approval}

Study NCT02204293 (Canakinumab for Treatment of Adult Onset Still's Disease) was approved by the PaulEhrlich-Institut (PEI) Germany (Postfach D-63207 Langen, Germany) on April 3, 2012 and the Ethic Committee Landesamt für Gesundheit und Soziales (LAGeSo) (Friedrichstrasse 16 in D-10969 Berlin, Germany).

\section{Abbreviations}

AOSD: adult-onset Still's disease; BL: baseline; IL: interleukin; SJIA: systemic juvenile idiopathic arthritis..

\section{Competing interests}

$A B$ is employee and shareholder of Novartis Pharma $A G$.

NB received royalties from Novartis, SOBI, Roche, UCB, MSD and AbbVie. EF has received speakers' honoraria and served as a consultant for Novartis, BMS, Pfizer, MSD, Lilly and Roche/Chugai.

GJ is employee of Novartis Pharma AG.

AM has received speakers' honoraria from Abbvie, Amgen, Biogenidec, BMS, Astellas, Boehringer, Italfarmaco, Janssen, Medlmmune, Novartis,

NovoNordisk, Pfizer, Sanofi, Roche, and Servier. Gaslini Hospital has received grants/research support from Abbott, Bristol-Myers Squibb, Francesco Angelini S.P.A., Glaxo Smith \& Kline, Janssen Biotech Inc, Novartis, Pfizer Inc, Roche, Sanofi Aventis, and Schwarz Biosciences $\mathrm{GmbH}$, and consulting fees from Bristol-Myers Squibb, Centocor Research \& Development, Glaxo Smith \& Kline, Novartis, Pfizer Inc, Roche, Sanofi Aventis, and Schwarz Biosciences GmbH, to support research activities of the Paediatric Rheumatology International Trials Organisation (PRINTO).

$\mathrm{NN}$ is employee of Novartis Institutes for Biomedical Research.

CS has no competing interests.

MW has received speakers' honoraria and served as a consultant for Novartis, Abbvie and Roche/Chugai.

JZ has no competing interests.

\section{Authors' contributions}

NN participated in the design of the analysis plan, coordinated the sample and data analysis, and helped draft the manuscript. $A B$ participated in the design of the analysis plan, performed the computational analysis of the gene expression data, and contributed to the manuscript writing. NB is a clinical investigator and contributed samples and patient data. NB, EF, AM, CS, and MW are principal investigators, and JZ was a study coordinator and made a substantial contribution to the logistic and performance of the biomarker substudy, for NCT02204293 (Canakinumab for Treatment of Adult Onset Still's Disease). GJ participated in the design of the analysis plan, coordinated the sample and data analysis, and helped draft the manuscript. All authors read and approved the final manuscript.

\section{Acknowledgements}

We thank the participating patients and investigators. This study was supported by Novartis Pharma AG. We thank BioScience Communications and Katia de Souza for providing editorial support on behalf of Novartis Pharma AG.

We thank Evert Luesink for assistance in adapting analysis scripts; Rahad Rasul for assistance with plotting of data; Martin Letzkus for his role in RNA extraction, and Nicole Hartmann for assistance with Affymetrix microarray hybridization. We also thank Anja Funkert for technical assistance.

\section{Author details}

${ }^{1}$ Novartis Institutes for BioMedical Research, Inc., 45 Sidney Street, Cambridge, MA 02139, USA. ${ }^{2}$ Novartis Institutes for Biomedical Research, $\mathrm{CH}-4002$ Basel, Switzerland. ${ }^{3}$ Department of Rheumatology and Clinical Immunology, Charité- Universitätsmedizin, Chariteplatz 1, 10117 Berlin, Germany. ${ }^{4}$ Division of Rheumatology, Department of Medicine, University of 
Heidelberg, Im Neuenheimer Feld 410, D-69120 Heidelberg, Germany. ${ }^{5}$ Klinik für Rheumatologie \& Klinische Immunologie, SJK - University Hospital Essen, Propsteistrasse 2, 45239 Essen, Germany. ${ }^{6}$ Division of Rheumatology and Clinical Immunology, Med. Klinik and Poliklinik IV, University of Munich, Pettenkoferstrasse 8a, 80336 Munich, Germany. ${ }^{7}$ University of Genoa and G Gaslini Institute, Head Pediatria II, Reumatologia, IRCCS G. Gaslini, Largo G. Gaslini 5, 616147 Genoa, Italy. ${ }^{8}$ Novartis Pharma AG, CH-4002 Basel, Switzerland.

Received: 14 July 2015 Accepted: 6 November 2015

Published online: 20 November 2015

\section{References}

1. Jamilloux Y, Gerfaud-Ventin M, Martinon F, Belot A, Henry T, Sève P. Pathogenesis of adult-onset Still's disease: new insights from the juvenile counterpart. Immunol Res. 2015;61:53-62.

2. Martini A. It is time to rethink juvenile idiopathic arthritis classification and nomenclature. Ann Rheum Dis. 2012;71:1437-9.

3. Fautrel B. Adult-onset Still disease. Best Pract Res Clin Rheumatol. 2008;22: 773-92.

4. Luthi F, Zufferey P, Hofer MF, So AK. "Adolescent-onset Still's disease": characteristics and outcome in comparison with adult-onset Still's disease. Clin Exp Rheumatol. 2002;20:427-30.

5. Uppal SS, Pande IR, Kumar A, Kailash S, Sekharan NG, Adya CM, et al. Adult onset Still's disease in northern India: comparison with juvenile onset Still's disease. Br J Rheumatol. 1995:34:429-34.

6. Still GF. On a form of chronic joint disease in children. Med Chir Trans. 1897; 80:47-60.9.

7. Frosch M, Metze D, Foell D, Vogl T, Sorg C, Sunderkötter C, et al. Early activation of cutaneous vessels and epithelial cells is characteristic of acute systemic onset juvenile idiopathic arthritis. Exp Dermatol. 2005;14:259-65.

8. Kim HA, An JM, Nam JY, Jeon JY, Suh CH. Serum S100A8/A9, but not follistatin-like protein 1 and interleukin 18, may be a useful biomarker of disease activity in adult-onset Still's disease. J Rheumatol. 2012;39:1399-406.

9. Chen D-Y, Chen Y-M, Lan J-L, Lin C-C, Chen H-H. Potential role of TH17 cells in the pathogenesis of adult-onset Still's disease. Rheumatology. 2010;49: 2305-12.

10. Kötter I, Wacker A, Koch S, Henes J, Richter C, Engel A, et al. Anakinra in patients with treatment-resistant adult-onset Still's disease: four case reports with serial cytokine measurements and a review of the literature. Semin Arthritis Rheum. 2007;37:189-97.

11. Avau A, Put K, Wouters CH, Matthys P. Cytokine balance and cytokine-driven natural killer cell dysfunction in systemic juvenile idiopathic arthritis. Cytokine Growth Factor Rev. 2015;26:35-45.

12. Félix FH, Leal LK, Fontenele JB. Cloak and dagger: the case for adult onset still disease and hemophagocytic lymphohistiocytosis. Rheumatol Int. 2009;29:973-4.

13. Ravelli A, Grom AA, Behrens EM, Cron RQ. Macrophage activation syndrome as part of systemic juvenile idiopathic arthritis: diagnosis, genetics, pathophysiology and treatment. Genes Immun. 2012;13:289-98.

14. Pascual V, Allantaz F, Arce E, Punaro M, Banchereau J. Role of interleukin-1 (IL-1) in the pathogenesis of systemic onset juvenile idiopathic arthritis and clinical response to IL-1 blockade. J Exp Med. 2005;201:1479-86.

15. Fall N, Barnes M, Thornton S, Luyrink L, Olson J, Ilowite NT, et al. Gene expression profiling of peripheral blood from patients with untreated newonset systemic juvenile idiopathic arthritis reveals molecular heterogeneity that may predict macrophage activation syndrome. Arthritis Rheum. 2007; 56:3793-804.

16. Ruperto N, Quartier P, Wulffraat N, Woo P, Ravelli A, Mouy R, et al. A phase II, multicenter, open-label study evaluating dosing and preliminary safety and efficacy of canakinumab in systemic juvenile idiopathic arthritis with active systemic features. Arthritis Rheum. 2012;64:557-67.

17. Ruperto N, Brunner HI, Quartier P, Constantin T, Wulfraat N, Horneff G, et al. Two randomized trials of canakinumab in systemic juvenile idiopathic arthritis. N Engl J Med. 2012;367:2396-406.

18. Kontzias A, Efthimiou P. The use of Canakinumab, a novel IL-1 $\beta$ long-acting inhibitor, in refractory adult-onset Still's disease. Semin Arthritis Rheum. 2012;42:201-5

19. Laskari K, Tzioufas AG, Moutsopoulos HM. Efficacy and long-term follow-up of IL-1R inhibitor anakinra in adults with Still's disease: a case-series study. Arthritis Res Ther. 2011;13:R91.
20. Efthimiou P, Kontzias A, Ward CM, Ogden NS. Adult-onset Still's disease: can recent advances in our understanding of its pathogenesis lead to targeted therapy? Nat Clin Pract Rheumatol. 2007;3:328-35.

21. Fitzgerald AA, Leclerca SA, Yan A, Hornik JE, Dinarello CA. Rapid responses to anakinra in patients with refractory adult-onset Still's disease. Arthritis Rheum. 2005;52:1794-803.

22. Petryna O, Cush JJ, Efthimiou P. IL-1 Trap rilonacept in refractory adult onset Still's disease. Ann Rheum Dis. 2012;71:2056-7.

23. Nordström D, Knight A, Luukkainen $R$, van Vollenhaven $R$, Rantalaiho V Kajalainen A, et al. Beneficial effect of interleukin 1 inhibition with anakinra in adult-onset Still's disease. An open, randomized, multicenter study. J Rheumatol. 2012;39:2008-11.

24. Naumann L, Feist E, Natusch A, Langen S, Krause A, Buttgereit F, et al. IL1receptor antagonist anakinra provides long-lasting efficacy in the treatment of refractory adult-onset Still's disease. Ann Rheum Dis. 2010;69:466-7.

25. Brachat A, Grom A, Wulffraat N, Brunner H, Quartier P, Brik R, et al. Changes in gene expression and inflammatory proteins in systemic juvenile idiopathic arthritis patients on canakinumab therapy. Ann Rheum Dis. 2014; 73(Suppl2):62.

26. ClinicalTrials.gov. NCT02204293. https://clinicaltrials.gov/ct2/show/NCT02204293. Accessed November 18, 2015.

27. Wu D, Lim E, Vaillant F, Asselin-Labat ML, Visvader JE, Smyth GK. ROAST: rotation gene set tests for complex microarray experiments. Bioinformatics. 2010;26:2176-82

\section{Submit your next manuscript to BioMed Central and take full advantage of:}

- Convenient online submission

- Thorough peer review

- No space constraints or color figure charges

- Immediate publication on acceptance

- Inclusion in PubMed, CAS, Scopus and Google Scholar

- Research which is freely available for redistribution

Submit your manuscript at www.biomedcentral.com/submit 